1 Doing the $\mathcal{D} \mu$ integration

$$
\begin{aligned}
\frac{1}{\sqrt{\operatorname{det} A^{-1}}} & =\frac{1}{\sqrt{\frac{1}{\operatorname{det} A}}} \\
& =\sqrt{\operatorname{det} A} \\
& =\sqrt{\operatorname{det}\left[-2 i\left(\mathcal{L}+\frac{\kappa}{\sqrt{\delta(\mathbf{0})}}\right)\right]} \\
& =\sqrt{\operatorname{det}(-2 i) \operatorname{det}\left(\mathcal{L}+\frac{\kappa}{\sqrt{\delta(\mathbf{0})}}\right)} \\
& \propto \exp \frac{1}{2} \operatorname{Tr} \ln \left(\mathcal{L}+\frac{\kappa}{\sqrt{\delta(\mathbf{0})}}\right) \\
& \propto \exp \left[\frac{1}{2} \operatorname{Tr} \ln \left(1+(\mathcal{L}-1)+\frac{\kappa}{\sqrt{\delta(\mathbf{0})}}\right)\right] \\
& \propto \exp \left[\frac{1}{2} \operatorname{Tr}\left(\mathcal{L}-1+\frac{\kappa}{\sqrt{\delta(\mathbf{0})}}-\frac{1}{2}\left((\mathcal{L}-1)+\frac{\kappa}{\sqrt{\delta(\mathbf{0})}}\right)^{2}\right)+\ldots\right] \\
& \propto \exp \left[\frac{1}{2} \operatorname{Tr} \mathcal{L}+\frac{\kappa \sqrt{\delta(\mathbf{0})}}{2}-\frac{1}{4} \operatorname{Tr}(\mathcal{L}-1)^{2}-\frac{1}{2} \operatorname{Tr}\left(\mathcal{L} \frac{\kappa}{\sqrt{\delta(\mathbf{0})}}\right)+\frac{1}{2} \frac{\kappa}{\left.\sqrt{\delta(\mathbf{0})} \delta(\mathbf{0})-\frac{1}{4} \kappa^{2} \frac{\delta(\mathbf{0})}{\delta(\mathbf{0})}\right]}\right. \\
& \propto \exp \left[\frac{\lambda(\mathbf{0})}{2}+\frac{\kappa \sqrt{\delta(\mathbf{0})}}{2}-\frac{1}{4}\left(\operatorname{Tr} \mathcal{L}^{2}-2 \operatorname{Tr} \mathcal{L}+\operatorname{Tr} 1\right)-\frac{1}{2} \frac{\kappa}{\sqrt{\delta(\mathbf{0})}} \lambda(\mathbf{0})+\frac{\kappa \sqrt{\delta(\mathbf{0})}}{2}-\frac{\kappa^{2}}{4}\right] \\
& \propto \exp \left[\frac{\lambda(\mathbf{0})}{2}+\kappa \sqrt{\delta(\mathbf{0})}-\frac{1}{4}\left(\frac{1}{4} \times 2\left\{\lambda^{t} \Sigma \lambda+\lambda^{t} \lambda\right\}-2 \lambda(\mathbf{0})\right)-\frac{1}{2} \frac{\kappa}{\sqrt{\delta(\mathbf{0})}} \lambda(\mathbf{0})-\frac{\kappa^{2}}{4}\right] \\
& \propto \exp \left[\lambda(\mathbf{0})+\kappa \sqrt{\delta(\mathbf{0})}-\frac{1}{8} \lambda^{t}(1+\Sigma) \lambda-\frac{1}{2} \frac{\kappa}{\sqrt{\delta(\mathbf{0})}} \lambda(\mathbf{0})-\frac{\kappa^{2}}{4}\right] \\
& \propto \exp \left[\lambda^{t} \delta+\kappa \sqrt{\delta(\mathbf{0})}-\frac{1}{8} \lambda^{t}(1+\Sigma) \lambda-\frac{1}{2} \frac{\kappa}{\sqrt{\delta(\mathbf{0})}} \lambda^{t} \delta-\frac{\kappa^{2}}{4}\right]
\end{aligned}
$$




$$
\begin{aligned}
& I=\int_{-\infty}^{\infty} d \kappa \exp \left[-\frac{1}{4} \kappa^{2}\right] \int \mathcal{D} \lambda \mathcal{D} \mu \exp \left[-\frac{1}{2} \mu^{t} A^{-1} \mu-i \lambda^{t}\left(Q-i \frac{\kappa}{2 \sqrt{\delta(\mathbf{0})}} \delta\right)\right] \exp \left[-i \mu^{t} \sqrt{N}\left(-1-\frac{i \kappa}{2 \sqrt{\delta(\mathbf{0})}}-i \lambda(\mathbf{0}) \frac{\sqrt{N}}{2 \delta(\mathbf{0})}\right)-\frac{1}{8} \lambda^{t}(1+\Sigma) \lambda\right] \times \\
& \exp \left[i \mu^{t} \frac{1}{A} \frac{M \sqrt{N}}{\delta(\mathbf{0})} \frac{1}{A} \mu\right] \exp \left[\frac{1}{2} \frac{\delta^{t}}{\delta \rho} \mathcal{K}^{-1} \frac{\delta}{\delta \rho}\right] E_{\mathbf{h}} E_{\mathbf{k}} E_{-\mathbf{h}-\mathbf{k}}[\rho]_{\rho=\varphi} \\
& \varphi=-i \mathcal{K}^{-1} \mu \\
& \mathcal{K}=-2 i\left(\mathcal{L}+\frac{\kappa}{\sqrt{\delta(\mathbf{0})}}+\frac{M \sqrt{N}}{\delta(\mathbf{0})}\right) \\
& =A-2 i \frac{M \sqrt{N}}{\delta(\mathbf{0})} \\
& A=-2 i\left(\mathcal{L}+\frac{\kappa}{\sqrt{\delta(\mathbf{0})}}\right) \\
& I=\int d \kappa \exp \left[-\frac{1}{4} \kappa^{2}\right] \int \mathcal{D} \lambda \sqrt{\operatorname{det} A} \exp \left[-i \lambda^{t}\left(Q-\frac{i \kappa}{2 \sqrt{\delta(\mathbf{0})}} \delta\right)-\frac{1}{8} \lambda^{t}(1+\Sigma) \lambda\right] \times \\
& \exp \left[-\frac{1}{2} \sqrt{N}\left(-1-\frac{i \kappa}{2 \sqrt{\delta(\mathbf{0})}}-i \lambda(\mathbf{0}) \frac{\sqrt{N}}{2 \delta(\mathbf{0})}\right) A \sqrt{N}\left(-1-\frac{i \kappa}{2 \sqrt{\delta(\mathbf{0})}}-i \lambda(\mathbf{0}) \frac{\sqrt{N}}{2 \delta(\mathbf{0})}\right)\right] \times \\
& \exp \left[\frac{1}{2} \frac{\delta^{t}}{\delta \mu} A \frac{\delta}{\delta \mu}\right]\left\{\exp \left[i \mu^{t} \frac{1}{A} \frac{M \sqrt{N}}{\delta(\mathbf{0})} \frac{1}{A} \mu\right] \exp \left[\frac{1}{2} \frac{\delta^{t}}{\delta \rho} \mathcal{K}^{-1} \frac{\delta}{\delta \rho}\right] E_{\mathbf{h}} E_{\mathbf{k}} E_{-\mathbf{h}-\mathbf{k}}[\rho]_{\rho=-i \mathcal{K}^{-1} \mu}\right\}_{\mu=i A \sqrt{N}\left(1+\frac{i \kappa}{2 \sqrt{\delta(\mathbf{0})}}+i \lambda(\mathbf{0}) \frac{\sqrt{N}}{2 \delta(\mathbf{0})}\right)} \\
& =\int d \kappa \exp \left[-\frac{1}{4} \kappa^{2}\right] \int \mathcal{D} \lambda \exp \left[-i \lambda^{t}\left(Q-\frac{i \kappa}{2 \sqrt{\delta(\mathbf{0})}} \delta\right)-\frac{1}{8} \lambda^{t}(1+\Sigma) \lambda+\lambda^{t} \delta+\kappa \sqrt{\delta(\mathbf{0})}-\frac{1}{8} \lambda^{t}(1+\Sigma) \lambda-\frac{1}{2} \frac{\kappa}{\sqrt{\delta(\mathbf{0})}} \lambda^{t} \delta-\frac{\kappa^{2}}{4}\right] \times \\
& \exp \left[\frac{1}{2} \sqrt{N}\left(-1-\frac{i \kappa}{2 \sqrt{\delta(\mathbf{0})}}-i \lambda(\mathbf{0}) \frac{\sqrt{N}}{2 \delta(\mathbf{0})}\right) A \sqrt{N}\left(-1-\frac{i \kappa}{2 \sqrt{\delta(\mathbf{0})}}-i \lambda(\mathbf{0}) \frac{\sqrt{N}}{2 \delta(\mathbf{0})}\right)\right] \times \\
& \exp \left[\frac{1}{2} \frac{\delta}{\delta \mu}^{t} A \frac{\delta}{\delta \mu}\right]\left\{\exp \left[i \mu^{t} \frac{1}{A} \frac{M \sqrt{N}}{\delta(\mathbf{0})} \frac{1}{A} \mu\right] \exp \left[\frac{1}{2} \frac{\delta}{\delta \rho}^{t} \mathcal{K}^{-1} \frac{\delta}{\delta \rho}\right] E_{\mathbf{h}} E_{\mathbf{k}} E_{-\mathbf{h}-\mathbf{k}}[\rho]_{\rho=-i \mathcal{K}^{-1} \mu}\right\}_{\mu=i A \sqrt{N}\left(1+\frac{i \kappa}{2 \sqrt{\delta(\mathbf{0})}}+i \lambda(\mathbf{0}) \frac{\sqrt{N}}{2 \delta(\mathbf{0})}\right)} \\
& =\int d \kappa \exp \left[-\frac{1}{2} \kappa^{2}+\kappa \sqrt{\delta(\mathbf{0})}\right] \int \mathcal{D} \lambda \exp \left[-i \lambda^{t}\left(Q+i \delta-\frac{i \kappa}{\sqrt{\delta(\mathbf{0})}} \delta\right)-\frac{1}{4} \lambda^{t}(1+\Sigma) \lambda+\lambda^{t} \delta\right] \times \\
& \exp \left[\frac{1}{2} \sqrt{N}\left(-1-\frac{i \kappa}{2 \sqrt{\delta(\mathbf{0})}}-i \lambda(\mathbf{0}) \frac{\sqrt{N}}{2 \delta(\mathbf{0})}\right) A \sqrt{N}\left(-1-\frac{i \kappa}{2 \sqrt{\delta(\mathbf{0})}}-i \lambda(\mathbf{0}) \frac{\sqrt{N}}{2 \delta(\mathbf{0})}\right)\right] \times \\
& \left.\exp \left[\frac{1}{2} \frac{\delta^{t}}{\delta \mu} A \frac{\delta}{\delta \mu}\right]\left\{\exp \left[i \mu^{t} \frac{1}{A} \frac{M \sqrt{N}}{\delta(\mathbf{0})} \frac{1}{A} \mu\right] \exp \left[\frac{1}{2} \frac{\delta^{t}}{\delta \rho} \mathcal{K}^{-1} \frac{\delta}{\delta \rho}\right] E_{\mathbf{h}} E_{\mathbf{k}} E_{-\mathbf{h}-\mathbf{k}}[\rho]_{\rho=-i \mathcal{K}^{-1} \mu}\right\}_{\mu=i A \sqrt{N}\left(1+\frac{i \kappa}{2 \sqrt{\delta(\mathbf{0})}}+i \lambda(\mathbf{0}) \frac{\sqrt{N}}{2 \delta(\mathbf{0})}\right.}\right)
\end{aligned}
$$




$$
\begin{aligned}
& =\int d \kappa \exp \left[-\frac{1}{2} \kappa^{2}+\kappa \sqrt{\delta(\mathbf{0})}\right] \int \mathcal{D} \lambda \exp \left[-i \lambda^{t}\left(Q+i \delta-\frac{i \kappa}{\sqrt{\delta(\mathbf{0})}} \delta\right)-\frac{1}{4} \lambda^{t}(1+\Sigma) \lambda+\lambda^{t} \delta\right] \times \\
& \exp \left[\left(i N\left(\int \lambda+\frac{\kappa}{\sqrt{\delta(\mathbf{0})}}\right)\right)\left(1+\frac{i \kappa}{2 \sqrt{\delta(\mathbf{0})}}+i \lambda(\mathbf{0}) \frac{\sqrt{N}}{2 \delta(\mathbf{0})}\right)^{2}\right] \times \\
& \exp \left[\frac{1}{2} \frac{\delta}{\delta \mu}^{t} A \frac{\delta}{\delta \mu}\right]\{\underbrace{\exp \left[i \mu^{t} \frac{1}{A} \frac{M \sqrt{N}}{\delta(\mathbf{0})} \frac{1}{A} \mu\right]}_{\mathcal{H}[\mu]} \underbrace{\exp \left[\frac{1}{2} \frac{\delta^{t}}{\delta \rho} \mathcal{K}^{-1} \frac{\delta}{\delta \rho}\right] E_{\mathbf{h}} E_{\mathbf{k}} E_{-\mathbf{h}-\mathbf{k}}[\rho]_{\rho=-i \mathcal{K}^{-1} \mu}}_{H[\mu]}\}_{\mu=i A \sqrt{N}\left(1+\frac{i \kappa}{2 \sqrt{\delta(\mathbf{0})}}+i \lambda(\mathbf{0}) \frac{\sqrt{N}}{2 \delta(\mathbf{0})}\right)} \\
& H[\mu]=E_{\mathbf{h}} E_{\mathbf{k}} E_{-\mathbf{h}-\mathbf{k}}[\rho]_{\rho=-i \mathcal{K}^{-1} \mu}-i \int d \mathbf{u} d \mathbf{v} \exp [2 \pi i(\mathbf{h} \cdot \mathbf{u}+\mathbf{k} \cdot \mathbf{v})]\left\{\int d \mathbf{z} d \mathbf{y}\left[\mathcal{K}_{\mathbf{z}, \mathbf{z}+\mathbf{u}}^{-1} \mathcal{K}_{\mathbf{z}+\mathbf{v}, \mathbf{y}}^{-1} \mu(\mathbf{y})+\mathcal{K}_{\mathbf{z}, \mathbf{z}+\mathbf{v}}^{-1} \mathcal{K}_{\mathbf{z}+\mathbf{u}, \mathbf{y}}^{-1} \mu(\mathbf{y})+\mathcal{K}_{\mathbf{z}+\mathbf{u}, \mathbf{z}+\mathbf{v}}^{-1} \mathcal{K}_{\mathbf{z}, \mathbf{y}}^{-1} \mu(\mathbf{y})\right]\right\}
\end{aligned}
$$

\section{Calculation of $L_{\mathrm{h}, \mathrm{k}}$.}

$\langle\mathbf{x} \mid \mathbf{h}\rangle \equiv \exp [-2 \pi i \mathbf{h} \cdot \mathbf{x}]$. Then $\langle\mathbf{h} \mid \mathbf{x}\rangle \equiv \overline{\langle\mathbf{x} \mid \mathbf{h}\rangle}=\exp [2 \pi i \mathbf{h} \cdot \mathbf{x}]=\langle-\mathbf{x} \mid \mathbf{h}\rangle=\langle\mathbf{x} \mid-\mathbf{h}\rangle$ 


$$
\begin{aligned}
& L_{\mathbf{h}, \mathbf{k}}=\int d \mathbf{x} d \mathbf{y}\langle\mathbf{h} \mid \mathbf{x}\rangle\langle\mathbf{x}|L| \mathbf{y}\rangle\langle\mathbf{y} \mid \mathbf{k}\rangle \\
& =\int d \mathbf{x} d \mathbf{y}\langle\mathbf{h} \mid \mathbf{x}\rangle L_{\mathbf{x}, \mathbf{y}}\langle\mathbf{y} \mid \mathbf{k}\rangle \\
& =\int d \mathbf{x} d \mathbf{y}\langle\mathbf{h} \mid \mathbf{x}\rangle \lambda(\mathbf{x}-\mathbf{y})\langle\mathbf{y} \mid \mathbf{k}\rangle \\
& =\int d \mathbf{x}\langle\mathbf{h} \mid \mathbf{x}\rangle \int-d \mathbf{u} \lambda(\mathbf{u})\langle\mathbf{y}-\mathbf{x}+\mathbf{x} \mid \mathbf{k}\rangle \quad \mathbf{u}=\mathbf{x}-\mathbf{y} \\
& =-\int d \mathbf{x}\langle\mathbf{h} \mid \mathbf{x}\rangle \int d \mathbf{u} \lambda(\mathbf{u})\langle-\mathbf{u}+\mathbf{x} \mid \mathbf{k}\rangle \\
& =-\int d \mathbf{x}\langle\mathbf{h} \mid \mathbf{x}\rangle\langle\mathbf{x} \mid \mathbf{k}\rangle \int d \mathbf{u} \lambda(\mathbf{u}) \underbrace{\langle-\mathbf{u} \mid \mathbf{k}\rangle}_{\langle\mathbf{k} \mid \mathbf{u}\rangle} \\
& =-\int d \mathbf{x}\langle\mathbf{h} \mid \mathbf{x}\rangle\langle\mathbf{x} \mid \mathbf{k}\rangle \int d \mathbf{u}\langle\mathbf{k} \mid \mathbf{u}\rangle\langle\mathbf{u} \mid \lambda\rangle \\
& =-\underbrace{\int d \mathbf{x}\langle\mathbf{h} \mid \mathbf{x}\rangle\langle\mathbf{x} \mid \mathbf{k}\rangle}_{\langle\mathbf{h} \mid \mathbf{k}\rangle}\langle\mathbf{k} \mid \lambda\rangle \\
& =-(2 \pi)^{3} \delta(\mathbf{h}-\mathbf{k}) \hat{\lambda}(\mathbf{k}) \\
& L_{\mathbf{h}, \mathbf{k}}^{t}=\int d \mathbf{x} d \mathbf{y}\langle\mathbf{h} \mid \mathbf{x}\rangle\left\langle\mathbf{x}\left|L^{t}\right| \mathbf{y}\right\rangle\langle\mathbf{y} \mid \mathbf{k}\rangle \\
& =\int d \mathbf{x} d \mathbf{y}\langle\mathbf{h} \mid \mathbf{x}\rangle L_{\mathbf{x}, \mathbf{y}}^{t}\langle\mathbf{y} \mid \mathbf{k}\rangle \\
& =\int d \mathbf{x} d \mathbf{y}\langle\mathbf{h} \mid \mathbf{x}\rangle \lambda(\mathbf{y}-\mathbf{x})\langle\mathbf{y} \mid \mathbf{k}\rangle \\
& =\int d \mathbf{x}\langle\mathbf{h} \mid \mathbf{x}\rangle \int d \mathbf{u} \lambda(\mathbf{u})\langle\mathbf{y}-\mathbf{x}+\mathbf{x} \mid \mathbf{k}\rangle \quad \mathbf{u}=\mathbf{y}-\mathbf{x} \\
& =\int d \mathbf{x}\langle\mathbf{h} \mid \mathbf{x}\rangle \int d \mathbf{u} \lambda(\mathbf{u})\langle\mathbf{u}+\mathbf{x} \mid \mathbf{k}\rangle \\
& =\int d \mathbf{x}\langle\mathbf{h} \mid \mathbf{x}\rangle\langle\mathbf{x} \mid \mathbf{k}\rangle \int d \mathbf{u} \lambda(\mathbf{u})\langle\mathbf{u} \mid \mathbf{k}\rangle \\
& =d \mathbf{x}\langle\mathbf{h} \mid \mathbf{x}\rangle\langle\mathbf{x} \mid \mathbf{k}\rangle \int d \mathbf{u}\langle-\mathbf{k} \mid \mathbf{u}\rangle\langle\mathbf{u} \mid \lambda\rangle \\
& =\langle\mathbf{h} \mid \mathbf{k}\rangle\langle-\mathbf{k} \mid \lambda\rangle \\
& =(2 \pi)^{3} \delta(\mathbf{h}-\mathbf{k}) \hat{\lambda}(-\mathbf{k})
\end{aligned}
$$

4 


$$
\begin{aligned}
\mathcal{L}_{\mathbf{h}, \mathbf{k}} & =\frac{1}{2}\left(L_{\mathbf{h}, \mathbf{k}}+L_{\mathbf{h}, \mathbf{k}}^{t}\right) \\
& =\frac{1}{2}(2 \pi)^{3} \delta(\mathbf{h}-\mathbf{k})[\hat{\lambda}(-\mathbf{h})-\hat{\lambda}(\mathbf{h})] \quad \mathcal{L}_{\mathbf{h}, \mathbf{k}} \text { is diagonal }
\end{aligned}
$$

When $\lambda$ is even: $\lambda(\mathbf{u})=\lambda(-\mathbf{u})$ then

$$
\begin{aligned}
\hat{\lambda}(-\mathbf{h}) & =\langle-\mathbf{h} \mid \lambda\rangle \\
& =\int d \mathbf{u}\langle-\mathbf{h} \mid \mathbf{u}\rangle\langle\mathbf{u} \mid \lambda\rangle \\
& =\int d \mathbf{u}\langle\mathbf{h} \mid-\mathbf{u}\rangle\langle\mathbf{u} \mid \lambda\rangle \\
& =-\int d \mathbf{u}\langle\mathbf{h} \mid \mathbf{u}\rangle\langle-\mathbf{u} \mid \lambda\rangle \\
& =-\int d \mathbf{u}\langle\mathbf{h} \mid \mathbf{u}\rangle\langle\mathbf{u} \mid \lambda\rangle \\
& =-\langle\mathbf{h} \mid \lambda\rangle \\
& \equiv-\hat{\lambda}(\mathbf{h}) \\
\text { Then } \mathcal{L}_{\mathbf{h}, \mathbf{k}} & =-(2 \pi)^{3} \delta(\mathbf{h}-\mathbf{k}) \hat{\lambda}(\mathbf{h})
\end{aligned}
$$

3 Final calculations. From now on we assume that $\lambda$ is even!

1. $\left.H_{1}^{0}[\mu]\right|_{\mu=A \alpha}=0$

Indeed

$$
\begin{aligned}
\left.\left.E_{\mathbf{h}} E_{\mathbf{k}} E_{-\mathbf{h}-\mathbf{k}}[\rho]\right|_{\rho=-i A^{-1} \mu}\right|_{\mu=A \alpha} & =\left.E_{\mathbf{h}} E_{\mathbf{k}} E_{-\mathbf{h}-\mathbf{k}}[\rho]\right|_{\rho=-i A^{-1} A \alpha} \\
& \propto \alpha^{3}(\underbrace{\int d \mathbf{u}<\mathbf{h} \mid \mathbf{u}>}_{=0})(\underbrace{\int d \mathbf{v}<\mathbf{k} \mid \mathbf{v}>}_{=0})(\underbrace{\int d \mathbf{z}\langle-\mathbf{h}-\mathbf{k} \mid \mathbf{z}\rangle}_{=0})=0
\end{aligned}
$$

2. $\left.H_{1}^{1}[\mu]\right|_{\mu=A \alpha}=0$.

$$
-i \int d \mathbf{u} d \mathbf{v} d \mathbf{z}<\mathbf{h}|\mathbf{u}><\mathbf{k}| \mathbf{v}>\langle-\mathbf{h}-\mathbf{k} \mid \mathbf{z}\rangle \int d \mathbf{s} A_{\mathbf{z}, \mathbf{u}}^{-1} \underbrace{\int d \mathbf{y} A_{\mathbf{v}, \mathbf{y}}^{-1} A_{\mathbf{y}, \mathbf{s}}}_{\delta(\mathbf{v}-\mathbf{s})} \alpha \propto(\underbrace{\int d \mathbf{v}<\mathbf{k} \mid \mathbf{v}>}_{=0}) \int d \mathbf{z} d \mathbf{u}<\mathbf{u} \mid \mathbf{h}>\langle-\mathbf{h}-\mathbf{k} \mid \mathbf{z}\rangle A_{\mathbf{z}, \mathbf{u}}^{-1}=0
$$


3. $H_{2}^{0}[\mu]_{\mu=A \alpha}=0$.

Indeed e.g.

$$
i \int d \mathbf{u} d \mathbf{v} d \mathbf{z} d \mathbf{x}_{1} d \mathbf{x}_{2} d \mathbf{x}_{3}<\mathbf{h}|\mathbf{u}><\mathbf{k}| \mathbf{v}><-\mathbf{h}-\mathbf{k} \mid \mathbf{z}>\mathcal{K}_{\mathbf{z}, \mathbf{x}_{1}}^{-1} \mu\left(\mathbf{x}_{1}\right) \mathcal{K}_{\mathbf{u}, \mathbf{x}_{2}}^{-1} \mu\left(\mathbf{x}_{2}\right) \mathcal{K}_{\mathbf{v}, \mathbf{x}_{3}}^{-1} \mu\left(\mathbf{x}_{3}\right)=0
$$

Indeed at least one of the $\mathcal{K}$ equals $A$, say $\mathcal{K}_{\mathbf{z}, \mathbf{x}_{1}}^{-1}=A_{\mathbf{z}, \mathbf{x}_{1}}^{-1}$. Then at $\mu=A \alpha$

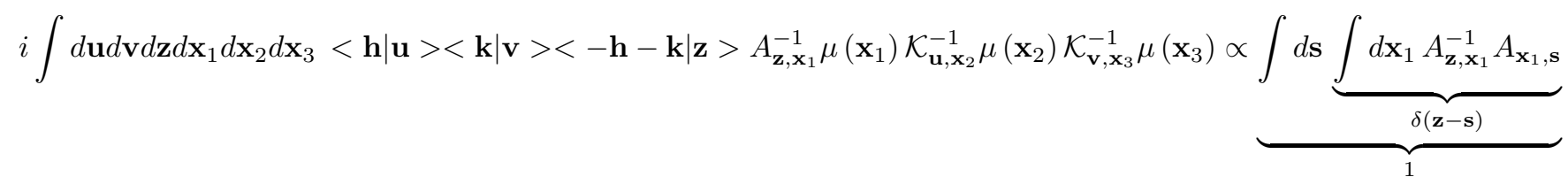

So this becomes 0 since

$$
\int d \mathbf{z}<-\mathbf{h}-\mathbf{k} \mid \mathbf{z}>=0
$$

4. $\mu(\mathbf{y})$ at $\mu=A \alpha$ is a constant (independent of $\mathbf{y}$ ) Indeed

$$
\begin{aligned}
\mu(\mathbf{y}) & =\alpha \int d \mathbf{s} A_{\mathbf{y}, \mathbf{s}} \\
& \propto \int d \mathbf{s} \int d \mathbf{p} d \mathbf{q}<\mathbf{y}|\mathbf{p}>\underbrace{\mathbf{p}|A| \mathbf{q}>}_{A(\mathbf{p}) \delta(\mathbf{p}-\mathbf{q})}<\mathbf{q}| \mathbf{s}> \\
& \propto \int d \mathbf{s} \int d \mathbf{p}<\mathbf{y}|\mathbf{p}>A(\mathbf{p})<\mathbf{p}| \mathbf{s}> \\
& \propto \int d \mathbf{p}<\mathbf{y} \mid \mathbf{p}>A(\mathbf{p}) \underbrace{\int d \mathbf{s}<\mathbf{p} \mid \mathbf{s}>}_{\delta(\mathbf{p})} \\
& \propto A(\mathbf{0})
\end{aligned}
$$

5. $\int d \mathbf{y} A_{\mathbf{w}, \mathbf{y}}^{-1}$ is a constant (independent of $\mathbf{w}$ ). Indeed

$$
\begin{aligned}
\int d \mathbf{y} A_{\mathbf{w}, \mathbf{y}}^{-1} & \propto \int d \mathbf{y} \int d \mathbf{p} d \mathbf{q}<\mathbf{w}|\mathbf{p}>\underbrace{<\mathbf{p}\left|A^{-1}\right| \mathbf{q}>}_{A^{-1}(\mathbf{p}) \delta(\mathbf{p}-\mathbf{q})}<\mathbf{q}| \mathbf{y}> \\
& \propto \int d \mathbf{p}<\mathbf{w} \mid \mathbf{p}>A^{-1}(\mathbf{p}) \underbrace{\int d \mathbf{y}<\mathbf{p} \mid \mathbf{y}>}_{\delta(\mathbf{p})} \\
& \propto A^{-1}(\mathbf{0})
\end{aligned}
$$


6. $\int d \mathbf{y} \mathcal{K}_{\mathbf{w}, \mathbf{y}}^{-1}$ is independent of $\mathbf{w}$

7. $H_{2}^{1}[\mu]_{\mu=A \alpha}=0$.

Indeed the $\delta(\mathbf{0})^{-1}$ (at $\mu=A \alpha$ ) part of

$$
-i \int d \mathbf{u} d \mathbf{v} d \mathbf{z}<\mathbf{h}|\mathbf{u}><\mathbf{k}| \mathbf{v}><-\mathbf{h}-\mathbf{k} \mid \mathbf{z}>\times\left[\int d \mathbf{y} \mathcal{K}_{\mathbf{z}, \mathbf{u}}^{-1} \mathcal{K}_{\mathbf{v}, \mathbf{y}}^{-1} \mu(\mathbf{y})+\int d \mathbf{y} \mathcal{K}_{\mathbf{z}, \mathbf{v}}^{-1} \mathcal{K}_{\mathbf{u}, \mathbf{y}}^{-1} \mu(\mathbf{y})+\int d \mathbf{z} d \mathbf{y} \mathcal{K}_{\mathbf{u}, \mathbf{v}}^{-1} \mathcal{K}_{\mathbf{z}, \mathbf{y}}^{-1} \mu(\mathbf{y})\right]
$$

gives 0 . Indeed

$\mathcal{K}^{-1}=A^{-1}+\frac{1}{\delta(\mathbf{0})} 2 i \sqrt{N} A^{-1} M A^{-1}+O\left(\delta(\mathbf{0})^{-2}\right)$. Consider the case $\mu=\mathcal{K} \alpha$. Then e.g.

$$
\int d \mathbf{y} \mathcal{K}_{\mathbf{z}, \mathbf{u}}^{-1} \mathcal{K}_{\mathbf{v}, \mathbf{y}}^{-1} \mu(\mathbf{y})=\int d \mathbf{y} \mathcal{K}_{\mathbf{z}, \mathbf{u}}^{-1} \underbrace{\int d \mathbf{s} \mathcal{K}_{\mathbf{v}, \mathbf{y}}^{-1} \mathcal{K}_{\mathbf{y}, \mathbf{s}}}_{1}
$$

and $\int d \mathbf{v}<\mathbf{k} \mid \mathbf{v}>=0$.

8. $\left.\frac{\delta}{\delta \mu}^{t} A \frac{\delta}{\delta \mu} H_{1}^{0}[\mu]\right|_{\mu=A \alpha}=0$. Indeed

one of the $\mu^{\prime}$ s in $H_{1}^{0}[\mu]$ survives the differentiation and then we have the reasoning of nr. 1 . Hence the contribution is at least of order $\delta(\mathbf{0})^{-1}$

9. $\left(H_{1}[\mu] \mathcal{H}_{1}[\mu]+H_{2}[\mu]\right)_{\mu=A \alpha}=0$ since $H_{1}[\mu]$ and $H_{2}[\mu]$ are 0 at $\mu=A \alpha$ (see above)

10. $\left.\frac{1}{2} \frac{\delta}{\delta \mu}^{t} A \frac{\delta}{\delta \mu}\left(H_{1}^{0}[\mu] \mathcal{H}_{1}[\mu]+H_{1}^{1}[\mu] \mathcal{H}_{1}[\mu]+H_{2}^{0}[\mu]\right)\right|_{\mu=A \alpha}=0$

11. $\frac{1}{8}\left(\frac{\delta}{\delta \mu}^{t} A \frac{\delta}{\delta \mu}^{2}\left(H_{1}^{0}[\mu] \mathcal{H}_{1}[\mu]+H_{2}^{0}[\mu]\right)_{\mu=A \alpha}=-\sqrt{N}\left(A_{\mathbf{z u}}^{-1} A_{\mathbf{u} v}^{-1}+A_{\mathbf{z v}}^{-1} A_{\mathbf{u} v}^{-1}+A_{\mathbf{z u}}^{-1} A_{z v}^{-1}\right)\right.$.

(a) $\left.\frac{1}{8}\left(\frac{\delta}{\delta \mu}^{t} A \frac{\delta}{\delta \mu}\right)^{2} \underbrace{H_{1}^{0}[\mu] \mathcal{H}_{1}[\mu]}_{\operatorname{deg} 6}\right|_{\mu=A \alpha}=0$

(b) But

$$
\begin{aligned}
\frac{1}{8}\left(\frac{\delta}{\delta \mu}^{t} A \frac{\delta}{\delta \mu}\right)^{2} \underbrace{H_{2}^{0}[\mu]}_{\operatorname{deg} 4} & =-\frac{\sqrt{N}}{2}\left(A_{\mathbf{z u}}^{-1} A_{\mathbf{u} v}^{-1}+A_{\mathbf{z v}}^{-1} A_{\mathbf{u} v}^{-1}+A_{\mathbf{z u}}^{-1} A_{z v}^{-1}+A_{\mathbf{z v}}^{-1} A_{z u}^{-1}+A_{\mathbf{v u}}^{-1} A_{z v}^{-1}+A_{\mathbf{u v}}^{-1} A_{z u}^{-1}\right) \\
& =-\sqrt{N}\left(A_{\mathbf{z u}}^{-1} A_{\mathbf{u} v}^{-1}+A_{\mathbf{z v}}^{-1} A_{\mathbf{u} v}^{-1}+A_{\mathbf{z u}}^{-1} A_{z v}^{-1}\right)
\end{aligned}
$$

Indeed

$$
\begin{aligned}
i \int d \mathbf{u} d \mathbf{v} d \mathbf{z} d \mathbf{x}_{1} d \mathbf{x}_{2} d \mathbf{x}_{3} & <\mathbf{h}|\mathbf{u}><\mathbf{k}| \mathbf{v}><-\mathbf{h}-\mathbf{k} \mid \mathbf{z}>\mathcal{K}_{\mathbf{z}, \mathbf{x}_{1}}^{-1} \mu\left(\mathbf{x}_{1}\right) \mathcal{K}_{\mathbf{u}, \mathbf{x}_{2}}^{-1} \mu\left(\mathbf{x}_{2}\right) \mathcal{K}_{\mathbf{v}, \mathbf{x}_{3}}^{-1} \mu\left(\mathbf{x}_{3}\right) \\
\mathcal{K}^{-1} & =A^{-1}+\frac{1}{\delta(\mathbf{0})} 2 i \sqrt{N} A^{-1} M A^{-1}
\end{aligned}
$$




$$
\begin{aligned}
& \frac{1}{8}\left(\frac{\delta^{t}}{\delta \mu} A \frac{\delta}{\delta \mu}\right)^{2} 2 i \sqrt{N}\left(A^{-1} M A^{-1}\right)_{\mathbf{z}, \mathbf{x}_{1}} \mu\left(\mathbf{x}_{1}\right) A_{\mathbf{u}, \mathbf{x}_{2}}^{-1} \mu\left(\mathbf{x}_{2}\right) A_{\mathbf{v}, \mathbf{x}_{3}}^{-1} \mu\left(\mathbf{x}_{3}\right) \\
& =-\frac{\sqrt{N}}{4}\left(\frac{\delta^{t}}{\delta \mu} A \frac{\delta}{\delta \mu}\right)\left(\frac{\delta^{t}}{\delta \mu} A \frac{\delta}{\delta \mu}\right)\left(A^{-1} M A^{-1}\right)_{\mathbf{z}, \mathbf{x}_{1}} \mu\left(\mathbf{x}_{1}\right) A_{\mathbf{u}, \mathbf{x}_{2}}^{-1} \mu\left(\mathbf{x}_{2}\right) A_{\mathbf{v}, \mathbf{x}_{3}}^{-1} \mu\left(\mathbf{x}_{3}\right) \\
& =-\frac{\sqrt{N}}{4}\left(\frac{\delta}{\delta \mu}^{t} A \frac{\delta}{\delta \mu}\right)\left(\frac{\delta}{\delta \mu}^{t} A \frac{\delta}{\delta \mu}\right) A_{\mathbf{z s}}^{-1} \mu(\mathbf{s}) A_{\mathbf{s x}_{1}}^{-1} \mu\left(\mathbf{x}_{1}\right) A_{\mathbf{u}, \mathbf{x}_{2}}^{-1} \mu\left(\mathbf{x}_{2}\right) A_{\mathbf{v}, \mathbf{x}_{3}}^{-1} \mu\left(\mathbf{x}_{3}\right) \\
& =-\frac{\sqrt{N}}{4}\left(\frac{\delta^{t}}{\delta \mu} A \frac{\delta}{\delta \mu}\right) A_{\mathbf{z s}}^{-1} A_{\mathbf{s}, \mathbf{x}_{1}} A_{\mathbf{s} \mathbf{x}_{1}}^{-1} A_{\mathbf{u}, \mathbf{x}_{2}}^{-1} \mu\left(\mathbf{x}_{2}\right) A_{\mathbf{v}, \mathbf{x}_{3}}^{-1} \mu\left(\mathbf{x}_{3}\right) \\
& =-\frac{\sqrt{N}}{4} A_{\mathbf{z s}}^{-1} \underbrace{A_{\mathbf{s}, \mathbf{x}_{1}} A_{\mathbf{s} \mathbf{x}_{1}}^{-1}}_{\delta(\mathbf{0})} \underbrace{A_{\mathbf{u}, \mathbf{x}_{2}}^{-1} A_{\mathbf{x}_{2}, \mathbf{x}_{3}}}_{\delta\left(\mathbf{u}-\mathbf{x}_{3}\right)} A_{\mathbf{v}, \mathbf{x}_{3}}^{-1} \\
& =-\frac{\sqrt{N} \delta(\mathbf{0})}{4} A_{\mathbf{z s}}^{-1} A_{\mathbf{v}, \mathbf{u}}^{-1} \\
& \Longrightarrow<-\mathbf{h}-\mathbf{k} \mid \mathbf{z}>\underbrace{\int d \mathbf{s} A_{\mathbf{z s}}^{-1}}_{C t e} \\
& =\int d \mathbf{z}<-\mathbf{h}-\mathbf{k} \mid \mathbf{z}>=0 \\
& \text { Indeed } \int \mathrm{d} \mathbf{s} A_{\mathbf{z s}}^{-1} \propto \int d \mathbf{s}<\mathbf{z}|\mathbf{p}>\underbrace{<\mathbf{p}\left|A^{-1}\right| \mathbf{q}>}_{A^{-1}(\mathbf{p}) \delta(\mathbf{p}-\mathbf{q})}<\mathbf{q}| \mathbf{s}> \\
& =<\mathbf{z} \mid \mathbf{p}>A^{-1}(\mathbf{p}) \underbrace{\int d \mathbf{s}<\mathbf{p} \mid \mathbf{s}>}_{\delta(\mathbf{p})} \\
& =A^{-1}(\mathbf{0})
\end{aligned}
$$


ii. Next

$$
\begin{gathered}
-\frac{\sqrt{N}}{4}\left(\frac{\delta}{\delta \mu}^{t} A \frac{\delta}{\delta \mu}\right)\left(\frac{\delta}{\delta \mu}^{t} A \frac{\delta}{\delta \mu}\right) A_{\mathbf{z s}}^{-1} \mu(\mathbf{s}) A_{\mathbf{s} \mathbf{x}_{1}}^{-1} \mu\left(\mathbf{x}_{1}\right) A_{\mathbf{u}, \mathbf{x}_{2}}^{-1} \mu\left(\mathbf{x}_{2}\right) A_{\mathbf{v}, \mathbf{x}_{3}}^{-1} \mu\left(\mathbf{x}_{3}\right) \\
=-\frac{\sqrt{N}}{4} A_{\mathbf{z s}}^{-1} A_{s, x_{2}} A_{\mathbf{s} \mathbf{x}_{1}}^{-1} A_{\mathbf{u}, \mathbf{x}_{2}}^{-1} \underbrace{A_{x_{1}, x_{3}} A_{\mathbf{v}, \mathbf{x}_{3}}^{-1}}_{\delta\left(v-x_{1}\right)} \\
=-\frac{\sqrt{N}}{4} A_{\mathbf{z s}}^{-1} A_{s, x_{2}} A_{\mathbf{s} v}^{-1} A_{\mathbf{u}, \mathbf{x}_{2}}^{-1} \\
=-\frac{\sqrt{N}}{4} A_{\mathbf{z s}}^{-1} A_{\mathbf{s} v}^{-1} \underbrace{A_{s, x_{2}} A_{\mathbf{u}, \mathbf{x}_{2}}^{-1}}_{\delta(s-u)} \\
=-\frac{\sqrt{N}}{4} A_{\mathbf{z u}}^{-1} A_{\mathbf{u} v}^{-1}
\end{gathered}
$$$$
\Longrightarrow \mathbf{2} \times-\frac{\sqrt{N}}{4} A_{\mathbf{z u}}^{-1} A_{\mathbf{u} v}^{-1}=-\frac{\sqrt{N}}{2} A_{\mathbf{z u}}^{-1} A_{\mathbf{u} v}^{-1}
$$

iii. also

$$
\begin{aligned}
&-\frac{\sqrt{N}}{4}\left(\frac{\delta}{\delta \mu}^{t} A \frac{\delta}{\delta \mu}\right)\left(\frac{\delta}{\delta \mu}^{t} A \frac{\delta}{\delta \mu}\right) A_{\mathbf{z s}}^{-1} \mu(\mathbf{s}) A_{\mathbf{s x}_{1}}^{-1} \mu\left(\mathbf{x}_{1}\right) A_{\mathbf{u}, \mathbf{x}_{2}}^{-1} \mu\left(\mathbf{x}_{2}\right) A_{\mathbf{v}, \mathbf{x}_{3}}^{-1} \mu\left(\mathbf{x}_{3}\right) \\
&=-\frac{\sqrt{N}}{4} A_{\mathbf{z s}}^{-1} A_{s x_{3}} A_{\mathbf{s x}_{1}}^{-1} \underbrace{A_{x_{1} x_{2}} A_{\mathbf{u}, \mathbf{x}_{2}}^{-1}}_{\delta\left(u-x_{1}\right)} A_{\mathbf{v}, \mathbf{x}_{3}}^{-1} \\
&=-\frac{\sqrt{N}}{4} A_{\mathbf{z}}^{-1} A_{s x_{3}} A_{\mathbf{s} u}^{-1} A_{\mathbf{v}, \mathbf{x}_{3}}^{-1} \\
&=-\frac{\sqrt{N}}{4} A_{\mathbf{z v}}^{-1} A_{v u}^{-1}
\end{aligned}
$$$$
\Longrightarrow \mathbf{2} \times-\frac{\sqrt{N}}{4} A_{\mathbf{z v}}^{-1} A_{\mathbf{u} v}^{-1}=-\frac{\sqrt{N}}{2} A_{\mathbf{z v}}^{-1} A_{\mathbf{u} v}^{-1}
$$

12. $\left.\frac{1}{8}\left(\frac{\delta}{\delta \mu}^{t} A \frac{\delta}{\delta \mu}\right)^{2} \underbrace{H_{1}^{1}[\mu] \mathcal{H}_{1}[\mu]}_{\operatorname{deg} 4}\right|_{\mu=A \alpha}=0$ 
(a) We have

$$
\begin{aligned}
& \frac{1}{8}\left(\frac{\delta}{\delta \mu}^{t} A \frac{\delta}{\delta \mu}\right)^{2}-i \int d \mathbf{u} d \mathbf{v} d \mathbf{z}<\mathbf{h}|\mathbf{u}><\mathbf{k}| \mathbf{v}><-\mathbf{h}-\mathbf{k} \mid z>\times \\
& {\left[\int d \mathbf{y} A_{\mathbf{z}, \mathbf{u}}^{-1} A_{\mathbf{v}, \mathbf{y}}^{-1} \mu(\mathbf{y})+\int d \mathbf{y} A_{\mathbf{z}, \mathbf{v}}^{-1} A_{\mathbf{u}, \mathbf{y}}^{-1} \mu(\mathbf{y})+\int d \mathbf{y} A_{\mathbf{u}, \mathbf{v}}^{-1} A_{\mathbf{z}, \mathbf{y}}^{-1} \mu(\mathbf{y})\right] i \sqrt{N} \mu^{t} \frac{1}{A} M \frac{1}{A} \mu} \\
& =\frac{\sqrt{N}}{8}\left(\frac{\delta}{\delta \mu}^{t} A \frac{\delta}{\delta \mu}\right)\left(\frac{\delta}{\delta \mu}^{t} A \frac{\delta}{\delta \mu}\right) \int d \mathbf{u} d \mathbf{v} d \mathbf{z}<\mathbf{h}|\mathbf{u}><\mathbf{k}| \mathbf{v}><-\mathbf{h}-\mathbf{k} \mid z>\times \\
& {\left[\int d \mathbf{y} A_{\mathbf{z}, \mathbf{u}}^{-1} A_{\mathbf{v}, \mathbf{y}}^{-1} \mu(\mathbf{y})+\int d \mathbf{y} A_{\mathbf{z}, \mathbf{v}}^{-1} A_{\mathbf{u}, \mathbf{y}}^{-1} \mu(\mathbf{y})+\int d \mathbf{y} A_{\mathbf{u}, \mathbf{v}}^{-1} A_{\mathbf{z}, \mathbf{y}}^{-1} \mu(\mathbf{y})\right] \int d s d t d p \mu(s) A_{s t}^{-1} \mu(t) A_{t p}^{-1} \mu(p)}
\end{aligned}
$$

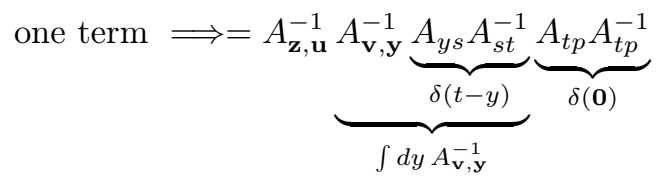

$$
\begin{aligned}
& \underbrace{\int d \mathbf{v}<\mathbf{k} \mid \mathbf{v}>\int d y A_{\mathbf{v}, \mathbf{y}}^{-1}}_{0}
\end{aligned}
$$

13. $\left.\frac{1}{48}\left(\frac{\delta}{\delta \mu}^{t} A \frac{\delta}{\delta \mu}\right)^{3}\left(H_{1}^{1}[\mu] \mathcal{H}_{1}[\mu]\right)\right|_{\mu=A \alpha}=-\frac{\sqrt{N}}{3} \int d \mathbf{u} d \mathbf{v} d \mathbf{z}<\mathbf{h}|\mathbf{u}><\mathbf{k}| \mathbf{v}><-\mathbf{h}-\mathbf{k} \mid \mathbf{z}>\left(A_{z u}^{-1} A_{u v}^{-1}+A_{z v}^{-1} A_{u v}^{-1}+A_{u z}^{-1} A_{z v}^{-1}\right)$

(a) Indeed

$$
\begin{gathered}
\frac{1}{48}\left(\frac{\delta^{t}}{\delta \mu} A \frac{\delta}{\delta \mu}\right)^{3} i \int d \mathbf{u} d \mathbf{v} d \mathbf{z} d \mathbf{x}_{1} d \mathbf{x}_{2} d \mathbf{x}_{3}<\mathbf{h}|\mathbf{u}><\mathbf{k}| \mathbf{v}><-\mathbf{h}-\mathbf{k} \mid \mathbf{z}>A_{\mathbf{z}, \mathbf{x}_{1}}^{-1} \mu\left(\mathbf{x}_{1}\right) A_{\mathbf{u}, \mathbf{x}_{2}}^{-1} \mu\left(\mathbf{x}_{2}\right) A_{\mathbf{v}, \mathbf{x}_{3}}^{-1} \mu\left(\mathbf{x}_{3}\right) \times \\
i \sqrt{N} \mu \mu^{t} \frac{1}{A} M \frac{1}{A} \mu \\
=-\frac{\sqrt{N}}{48} \int d \mathbf{u} d \mathbf{v} d \mathbf{z} d \mathbf{x}_{1} d \mathbf{x}_{2} d \mathbf{x}_{3}<\mathbf{h}|\mathbf{u}><\mathbf{k}| \mathbf{v}><-\mathbf{h}-\mathbf{k} \mid \mathbf{z}>\left(\frac{\delta^{t}}{\delta \mu} A \frac{\delta}{\delta \mu}\right)\left(\frac{\delta}{\delta \mu} A \frac{\delta}{\delta \mu}\right)\left(\frac{\delta}{\delta \mu} A \frac{\delta}{\delta \mu}\right) \times \\
A_{\mathbf{z}, \mathbf{x}_{1}}^{-1} \mu\left(\mathbf{x}_{1}\right) A_{\mathbf{u}, \mathbf{x}_{2}}^{-1} \mu\left(\mathbf{x}_{2}\right) A_{\mathbf{v}, \mathbf{x}_{3}}^{-1} \mu\left(\mathbf{x}_{3}\right) \int d s d t d p \mu(s) A_{s t}^{-1} \mu(t) A_{t p}^{-1} \mu(p) \\
\Longrightarrow=A_{\mathbf{z}, \mathbf{x}_{1}}^{-1} A_{x_{1} s} A_{\mathbf{u}, \mathbf{x}_{2}}^{-1} A_{x_{2} t} A_{\mathbf{v}, \mathbf{x}_{3}}^{-1} A_{x_{3} p} A_{s t}^{-1} A_{t p}^{-1} \\
=\delta(z-s) \delta(u-t) \delta(v-p) A_{s t}^{-1} A_{t p}^{-1} \quad \\
=-\frac{\sqrt{N}}{48} \int d \mathbf{u} d \mathbf{v} d \mathbf{z}<\mathbf{h}|\mathbf{u}><\mathbf{k}| \mathbf{v}><-\mathbf{h}-\mathbf{k} \mid \mathbf{z}>A_{z u}^{-1} A_{u v}^{-1}
\end{gathered}
$$


Thus

$$
\underbrace{6 \times 2 \times\left(-\frac{\sqrt{N}}{48}\right)}_{-\frac{\sqrt{N}}{3}} \int d \mathbf{u} d \mathbf{v} d \mathbf{z}<\mathbf{h}|\mathbf{u}><\mathbf{k}| \mathbf{v}><-\mathbf{h}-\mathbf{k} \mid \mathbf{z}>\left(A_{z u}^{-1} A_{u v}^{-1}+A_{z v}^{-1} A_{u v}^{-1}+A_{u z}^{-1} A_{z v}^{-1}\right)
$$

(b) Indeed e.g.

$$
\begin{aligned}
& \frac{1}{48}\left(\frac{\delta^{t}}{\delta \mu} A \frac{\delta}{\delta \mu}\right)^{3} i \int d \mathbf{u} d \mathbf{v} d \mathbf{z} d \mathbf{x}_{1} d \mathbf{x}_{2} d \mathbf{x}_{3}<\mathbf{h}|\mathbf{u}><\mathbf{k}| \mathbf{v}><-\mathbf{h}-\mathbf{k} \mid \mathbf{z}>A_{\mathbf{z}, \mathbf{x}_{1}}^{-1} \mu\left(\mathbf{x}_{1}\right) A_{\mathbf{u}, \mathbf{x}_{2}}^{-1} \mu\left(\mathbf{x}_{2}\right) A_{\mathbf{v}, \mathbf{x}_{3}}^{-1} \mu\left(\mathbf{x}_{3}\right) \times \\
& i \sqrt{N} \mu^{t} \frac{1}{A} M \frac{1}{A} \mu \\
& =-\frac{\sqrt{N}}{48} \int d \mathbf{u} d \mathbf{v} d \mathbf{z} d \mathbf{x}_{1} d \mathbf{x}_{2} d \mathbf{x}_{3}<\mathbf{h}|\mathbf{u}><\mathbf{k}| \mathbf{v}><-\mathbf{h}-\mathbf{k} \mid \mathbf{z}>\left(\frac{\delta}{\delta \mu}^{t} A \frac{\delta}{\delta \mu}\right)\left(\frac{\delta}{\delta \mu}^{t} A \frac{\delta}{\delta \mu}\right)\left(\frac{\delta}{\delta \mu}^{t} A \frac{\delta}{\delta \mu}\right) \times \\
& A_{\mathbf{z}, \mathbf{x}_{1}}^{-1} \mu\left(\mathbf{x}_{1}\right) A_{\mathbf{u}, \mathbf{x}_{2}}^{-1} \mu\left(\mathbf{x}_{2}\right) A_{\mathbf{v}, \mathbf{x}_{3}}^{-1} \mu\left(\mathbf{x}_{3}\right) \int d s d t d p \mu(s) A_{s t}^{-1} \mu(t) A_{t p}^{-1} \mu(p) \\
& \Longrightarrow=\underbrace{A_{\mathbf{z}, \mathbf{x}_{1}}^{-1} A_{x_{1} x_{2}}}_{\delta\left(z-x_{2}\right)} A_{\mathbf{u}, \mathbf{x}_{2}}^{-1} \underbrace{A_{\mathbf{v}, \mathbf{x}_{3}}^{-1} A_{x_{3} s}}_{\delta(v-s)} A_{s t}^{-1} \underbrace{A_{t p} A_{t p}^{-1}}_{\delta(\mathbf{0})} \\
& \Longrightarrow=\delta(\mathbf{0}) A_{\mathbf{u}, \mathbf{z}}^{-1} \int d v<\mathbf{k} \mid \mathbf{v}>\underbrace{\int \underbrace{\int d t A_{v t}^{-1}}_{\neq f(v)}}
\end{aligned}
$$

